\title{
Atomic structure of human sapovirus capsid by single particle cryo-electron microscopy
}

Naoyuki Miyazaki ${ }^{1}$, Kosuke Murakami $^{2}$, Tomoichiro Oka ${ }^{2}$, Motohiro Miki ${ }^{3}$, Kenji Iwasaki ${ }^{1}$,

Kazuhiko Katayama ${ }^{2,4}$, Kazuyoshi Murata ${ }^{5,6 *}$

${ }^{1}$ Life Science Center for Survival Dynamics, Tsukuba Advanced Research Alliance, University

of Tsukuba, 1-1-1 Tennodai, Tsukuba, Ibaraki, 305-8777, Japan

${ }^{2}$ Department of Virology II, National Institute of Infectious Diseases, Tokyo, Japan

${ }^{3}$ Vaccine \& Biomedicine Dept. Life Innovation Research Institute Denka Innovation Center,

Denka Co., Ltd., 3-5-1 Asahi-Machi Machida-City Tokyo, 194-8560, Japan

${ }^{4}$ Laboratory of Viral Infection I, Department of Infection Control and Immunology, Ōmura

Satoshi Memorial Institute \& Graduate School of Infection Control Sciences, Kitasato University,

Tokyo, 108-8641, Japan

${ }^{5}$ National Institute for Physiological Sciences, 38 Nishigonaka, Myodaiji, Okazaki, Aichi

444-8585, Japan

${ }^{6}$ Exploratory Research Center on Life and Living Systems (ExCELLS), National Institutes of

Natural Sciences, Okazaki, Aichi, Japan

*Correspondence: kazum@nips.ac.jp (Ka.M.) 


\section{Summary}

2 Sapovirus is a cause of acute gastroenteritis in humans and animals. Infants and younger children

3 have the greatest disease burden. Although it shares many similarities with norovirus, the lack of

4 detailed structural information has hampered the development of vaccines and therapeutics. Here,

5 we investigated the human sapovirus VLP by single particle cryo-electron microscopy and are

6 the first to report the atomic structure of the capsid at $2.9 \AA$ resolution. The atomic model

7 revealed the domain interactions of the capsid protein and functionally important amino acid

8 residues. The extended loop from the P1 subdomain was involved in interactions in the P2

9 domain, forming unique arch-like dimeric protrusions of capsid proteins. All hypervariable

10 regions that are important candidates for immune response or receptor binding, formed a large

11 cluster at the top of the $\mathrm{P}$ domain. These results pave the way for developing vaccines, antiviral

12 drugs, and diagnostic systems for this infectious disease.

\section{Keywords}

15 Sapovirus, Caliciviridae, capsid structure, cryo-electron microscopy, single particle analysis,

16 near-atomic resolution 
Introduction

19 Sapovirus ( $\mathrm{SaV}$ ) belongs to the Caliciviridae family and is well known to cause acute

20 gastroenteritis in humans as well as animals. Human $\mathrm{SaV}(\mathrm{HuSaV})$ contains a positive-sense

21 single-stranded RNA genome of approximately 7.1 to $7.5 \mathrm{~kb}$ in length (Green et al., 2001) and is

22 divided into four genogroups (GI, GII, GVI, and GV), although animal SaVs are further diverged

23 and divided into 19 genogroups (Farkas et al., 2004; Yinda et al. 2017; Li et al., 2018). The viral

24 genome consists of two or three open reading frames (ORFs). ORF1 encodes a polyprotein that

25 undergoes proteolytic cleavage to form non-structural proteins and a major capsid protein VP1

26 (viral protein 1). The major capsid protein VP1 is solely responsible for most capsid-related

27 functions, such as assembly, host interactions, and immunogenicity. ORF2 encodes a minor

28 structural protein, VP2. In the case of a feline calicivirus (FCV), a member of genus Vesivirus,

29 the minor structural protein VP2 forms a large dodecameric portal-like assembly at a unique

30 three-fold axis of icosahedral symmetry after receptor engagement (Conley et al., 2019), which

31 likely functions as a channel for genome release from the capsid. ORF3 encodes a small basic

32 protein of unknown function (Clarke and Lambden, 2000; Atmar and Estes, 2001). Compared

33 with well-characterized norovirus and vesivirus, there are limited studies on $\mathrm{SaV}$. In fact, the SaV

34 structure has been reported only at low and intermediate resolutions (Chen et al., 2004; Miyazaki

35 et al., 2016), and the atomic structure of the SaV capsid has not yet been elucidated. The SaV

36 belongs to a different genus from the well-characterized caliciviruses and has different host

37 specificity and immunogenicity. In addition, an understanding of replication strategies, 
38 pathogenesis, and immunogenicity of $\mathrm{SaV}$ have also been hampered due to the lack of a

39 sufficient viral replication system, such as the actual target cells in the host, until the recent

40 establishment of the SaV cultivation system (Takagi et al., 2020).

41 The Caliciviridae family is currently classified into eleven established genera: Bavovirus,

42 Lagovirus, Minovirus, Nacovirus, Nebovirus, Norovirus, Recovirus, Salovirus, Sapovirus,

43 Valovirus, and Vesivirus (Vinjé et al., 2019). The atomic structures of calicivirus VLPs or virions

44 in three established genera have been determined for Norwalk virus (NV; in genus Norovirus),

45 rabbit hemorrhagic disease virus (RHDV; in genus Lagovirus), San Miguel sea lion virus

46 (SMSV; in genus Vesivirus), and FCV (in genus Vesivirus), while atomic structures in other

47 genera, including Sapovirus, remain unknown (Prasad et al., 1999; Chen et al., 2006; Ossiboff et

48 al., 2010; Wang et al., 2013; Song et al., 2020). The calicivirus virions have a mostly conserved

49 capsid shell, which is composed of 180 copies of VP1 arranged in a T=3 icosahedral symmetry.

50 The VP1 proteins are designated A, B, and C in the icosahedral asymmetric unit according to

51 their positions, which form quasi-equivalent A/B and C/C dimers (Prasad et al., 1999; Chen et al.,

52 2006; Ossiboff et al., 2010). Each VP1 capsid monomer contains two principal domains, shell

53 (S) and protrusion (P) domains, with an N-terminal arm located inside the capsid shell. The S

54 domains represent the most conserved region of the amino acid sequence among caliciviruses,

55 and have a classical eight-stranded $\beta$ sandwich, which has been commonly found in $\mathrm{T}=3$

56 icosahedral viruses (Rossmann and Johnson, 1989). The fundamental function of the S domains

57 is to form a contiguous icosahedral capsid shell responsible for the protection of their viral 
58 genome from the outer environment. In contrast, structures and amino acid sequences of the $\mathrm{P}$

59 domain are rather variable among caliciviruses because this domain is involved in virus-host

60 interactions and immunogenicity. Indeed, the sizes of the P2 domains are considerably different

61 between Norovirus and Vesivirus, which are 127 and 176 amino acid residues, respectively

62 (Prasad et al., 1999; Chen et al., 2006). The P domains form protrusions on the capsid shell

63 composed of the $\mathrm{S}$ domains and each $\mathrm{P}$ domain can be further divided into two subdomains

64 called P1 and P2. In spite of the little sequence conservation, protein folds of the P1 and P2

65 subdomains are conserved among caliciviruses, but unique to other viruses except for hepatitis E

66 virus (HEV: Yamashita et al., 2009; Guu et al., 2009; Xing et al., 2010). The relative orientation

67 between S-P1-P2 domains shows inter-genus variations (rarely including intra-genus variations).

68 For example, only the P2 domain is involved in the dimeric interactions in Vesivirus, while both

69 the P1 and P2 domains participate in the dimeric interactions in Norovirus. Because of the

70 inter-genus diversities, we are eager to elucidate the atomic structures of other genera, including

71 Sapovirus, in order to understand their immunogenicity and virus-host interactions in more

72 detail.

73 Here, we determined the capsid structure of a HuSaV virus-like particle (HuSaV-VLP) at 2.9

74 Å resolution by single particle cryo-electron microscopy (cryo-EM), and successfully built an

75 atomic model of the capsid. The atomic model revealed the domain boundary and the

76 functionally important amino acid residues in the capsid protein, 1) the unique arch-like dimeric

77 protrusion on the capsid surface provides hints for a stable construct design for vaccine 
78 development, and 2) the detailed structure of the large hypervariable region cluster at the top of

79 the $\mathrm{P}$ domain accelerates the development of vaccines and antivirals.

\section{Results and Discussion}

84 The capsid protein VP1 of HuSaV from the Nichinan strain in genogroup I (GI. Nichinan;

85 Iwakiri et al., 2009) was expressed in a baculovirus expression system, and the self-assembled

86 and secreted HuSaV-VLPs from the cells were purified from the culture medium. The

87 three-dimensional (3D) structure was determined at $2.9 \AA$ resolution by cryo-EM single-particle

88 analysis (Figures $1, \mathrm{~S} 1$, and $\mathrm{S} 2$ ). The cryo-EM map clearly shows a $\mathrm{T}=3$ icosahedral symmetry

89 with 90 protrusions, composed of 180 copies of the VP1 protein in total, distributed along the

90 icosahedral 2-fold axes (C/C dimers) and quasi 2-fold axes (A/B dimers) on the surface (Figures

91 1A and S2A). The bulky side chains are clearly resolved in the cryo-EM, and the atomic models

92 are unambiguously built for the VP1 proteins (Figures 1B and S1E). The cryo-EM map allowed

93 atomic modeling of residues 38-554 for subunit $\mathrm{A}$, residues $21-554$ for subunit $\mathrm{B}$, and residues

94 21-554 for subunit C, except for one disordered loop (residues 380-384) for all subunits (Figure

95 2). The icosahedrally independent $\mathrm{A} / \mathrm{B}$ and $\mathrm{C} / \mathrm{C}$ dimers show slightly different conformations,

96 mainly between S-domains in each dimer, which are the bent and flat conformations, respectively,

97 as in other $\mathrm{T}=3$ viruses (Figure $\mathrm{S} 3$ ). The $\beta \mathrm{I}-\beta \mathrm{A}^{\prime}$ loop between the $\mathrm{S}$-domain and $\mathrm{P}$-domain, 
98 containing a $\beta$-turn (residues 231-234), undergoes a hinge-like motion to adapt the two

99 conformers (red asterisks in Figure S3). In addition, N-terminal regions of residues 21-37 in the

100 B- and C-subunits extend underneath the capsid shell and interact with the neighboring subunits

101 around icosahedral 3-fold axes (Figure 3A), although the N-terminal region is disordered in the

102 A-subunit, and no N-terminal network is observed around icosahedral 5-fold axes. In particular,

103 residues A25-T26 form a short inter-subunit $\beta$-sheet with F154-V155 in the adjacent subunit

104 (Figure 3B). Therefore, the N-terminal network around the icosahedral 3-fold axes likely

105 stabilized the hexameric units in the capsid.

\section{The structure of the major capsid VP1 protein of HuSaV}

108 The overall structure of the major capsid VP1 protein of HuSaV from GI. Nichinan

109 comprises two principal domains: S (residues 69-232) and P (residues 233-554) domains, with

110 an N-terminal arm (residues 21-68 in subunit B and C; residues 38-68 in subunit A) inside the

111 capsid shell (Figure 2). The P domain is further divided into two subdomains, P1 (233-281 and

112 residues 444-554) and P2 (residues 282-443) subdomains. These domain boundaries are

113 consistent with those from previous result based on the homology model built with an $8.5-\AA$

114 resolution cryo-EM map (Miyazaki et al., 2016). We found a disordered region (residues 381-

115384 ) in the P2 subdomain, which is located on the exterior surface of the viral particle (dashed

116 circles in Figure $2 \mathrm{~B}$ ), and the $\beta \mathrm{D}$ " $-\beta E$ " loop, including the disordered region, which is one of the

117 most valuable amino acid sequences among the HuSaVs (Figure 4). 
119 (residues 281-283) (Oka et al, 2015), indicated by red and black asterisks above sequences in

120 Figure 4, respectively. The former "PPG" motif exists in a $\beta E-\beta F$ loop and is a part of $\beta$-turn

121 (PPGV) just following a $\beta E$-strand in the S-domain, which is not exposed to the viral surface and

122 is located at the inter-domain interface between the S-domains (Figures 5A and 5C). The loop

123 after the $\beta$-turn interacts with the P1-subdomain (black arrowhead in Figure 5A). These

124 observations suggest that the conserved "PPG" motif forming the $\beta$-turn is involved in protein

125 folding, dimer formation, and assembly into the particle. The latter "GWS" motif is located at the

126 domain boundary between the P1 and P2 subdomains (Figure 5A). The "GWS" motif in the

$127 \beta \mathrm{A}^{\prime}-\beta \mathrm{A}^{\prime}$ loop is not exposed to the viral surface as in the motif "PPG" suggesting that the motif

128 is not involved in receptor binding, particle formation, or stability because W282 in the motif is

129 inserted into the P2 subdomain and forms a hydrophobic core with L322, I393, and M442 in the

130 P2 subdomains (Figure 5B).

133 The $\mathrm{P}$ domain of $\mathrm{HuSaV}$ forms a dimer on the surface of virus particles, and the inter-dimer

134 interaction occurs in the most exterior region of the dimer (Figures 1B and 6A), and the

135 inter-dimer orientation is similar to that of SMSV and FCV (Chen et al., 2006; Ossiboff et al.,

136 2010) as in the previous structure analysis at an 8- $\AA$ resolution (Miyazaki et al., 2016). In this

137 study, the high-resolution structure at $2.9 \AA$ resolution revealed the amino acid residues involved 
138 in the interaction between the P domains to build a stable P domain dimer. Unexpectedly, in

139 addition to the residues in the P2 subdomain, the residues in the P1 subdomain are involved in

140 the interaction between the P domains (Figure 6A). The $\beta B^{\prime}-\alpha 4$ loop (residues 465-469) in the

$141 \mathrm{P} 1$ subdomain and the $\beta C$ " $\beta \mathrm{D}$ " loop in the P2 subdomain are mainly involved in the interaction

142 between the P domains. Hydrophobic residues are present throughout the binding interface,

143 representing the hydrophobic interactions that appear to be dominant for the dimerization (Figure

144 6B), although some hydrophilic interactions are also observed, for example, between the $\beta B^{\prime}-\alpha 4$

145 loop and the $\beta C$ " $-\beta \mathrm{D}$ " loop. We calculated and compared buried surface areas between the P

146 domains of caliciviruses. The buried surface area between the $\mathrm{P}$ domains of HuSaV is $1.1 \times 10^{3}$

$147 \AA^{2}$, which is significantly smaller than those of other viruses $\left(1.5-1.7 \times 10^{3} \AA^{2}\right)$ (Figure 6C).

148 These results suggest that $\mathrm{HuSaV}$ shows a unique arch-like dimeric protrusion in Caliciviridae.

149 Furthermore, additional mechanisms may be required to stabilize the construct when the P

150 domain dimer is used as a vaccine antigen.

\section{Immune responses of HuSaVs}

153 To examine the immune reactivity of $\mathrm{HuSaVs}$, we analyzed the conserved sequences in the

154 structure (Figure 7). When the amino acid conservation across the human GI, GII, GIV, and GV

$155 \mathrm{SaVs}$, listed in Figure 4, are mapped on the 3D structure of VP1, the amino acid residues in the

156 P2 subdomain on the viral surface are extremely diverged, while those in the S and P1

157 subdomains are highly and intermediately conserved, respectively (Figure 7A). Furthermore, the 
158 primary sequence comparison shows that there are various insertions and deletions among the

$159 \mathrm{HuSaV}$ stains in the P2 subdomains (Figure 4). In particular, residues 294-306 in $\beta$ A'- $\beta$ B',

$160337-347$ in $\beta B^{\prime \prime}-\beta C$ ", 375-388 in $\beta D^{\prime \prime}-\beta E$ " (including a disordered region, 380-384), and 403-

161417 in $\beta E$ " $-\beta F$ " show significant sequence variabilities between the HuSaVs, which are

162 designated as hypervariable region 1 (HVR1), HVR2, HVR3, and HVR4, respectively (Figure 4).

163 These regions form a large cluster at the top of the P domain (Figure 7B). Generally, it is

164 believed that the P domain projected from the viral surface is mainly involved in immune

165 responses in caliciviruses, and the diverged amino acid sequences located on the most exterior

166 surface are responsible for evading the host's immune system. For instance, the neutralization

167 antigens of FCV existing in the HVR of residues 408-529 (Matsuura Y et al., 2001; Tohya et al.,

168 1991) are exclusively located in the P domain on the viral surface (Chen et al., 2006). HuSaV has

169 a large cluster consisting of four HVRs on the viral surface, suggesting that the viral strategy

170 against the host immune system, proposed by other caliciviruses, can be strongly applied to

$171 \mathrm{HuSaV}$.

172 Genogroup-specific and genotype-specific monoclonal antibodies (mAbs) have been

173 identified in a previous study (Kitamoto et al., 2012), reflecting their sequence diversity on the

174 viral surface. However, in spite of the sequence diversity, fully cross-reactive mAbs to

175 heterologous human genogroups and genotypes have also been found. These facts suggest that

$176 \mathrm{SaV}$ capsid proteins have at least one epitope common to human GI, GII, GIV, and GV

177 genogroups (Kitamoto et al., 2012). To examine the common epitope, we plotted the fully 
178 conserved amino acid residues (Figure 4) used in a previous immunological study (Kitamoto et

179 al., 2012) on the molecular surface of VP1. The fully conserved amino acid residues accessible

180 by the mAbs are limited to the $\mathrm{S}$ domain located in the region of the depression around the five-

181 or six-fold axes on the viral surface (orange circle in Figure 7C). Therefore, the result suggests

182 that one of the cross-reactive mAbs for all $\mathrm{HuSaVs}$ recognizes the $\mathrm{S}$ domain instead of the $\mathrm{P}$

183 domain.

184 Next, we examined epitopes recognized by mAbs specific to genogroups (GI, GII, and GIV)

185 (Figure S4). In the case of GI and GII, the amino acid residues conserved in each genogroup

186 form clusters in the P domain on the viral surface (magenta circles in Figures S4D-E) but not in

187 the S domain, suggesting that the GI- and GII-specific mAbs recognize the P2 subdomain. In

188 contrast, we found several clusters entirely in the P domain of GIV (Figures S4C and S4F).

189 Therefore, the GIV-specific mAbs also likely bind to the P domain. However, we found clusters

190 in the S domain (orange circle in Figure S4F), and therefore the possibility that the GIV-specific

191 mAbs recognize the S domain cannot be completely excluded.

192 Genotype-specific mAbs recognize amino acid residues that are not conserved even within

193 the genogroups. Many such amino acid residues are found in HVR1 to HVR4 on the viral

194 surface, as described above (Figures 4 and 7A-B) as well as in the $\beta F^{\prime \prime}-\beta B$ ' loop. Therefore, it is

195 considered that the highly variable regions are recognized by genotype-specific mAbs.

\section{Host specificity of HuSaV}


199 host recognition mechanism remains unknown. Porcine sapovirus (PoSaV) Cowden strain in a

200 genogroup GIII is the only culturable sapovirus that has been adapted to tissue culture-adapted

201 mutations (Lu et al., 2016; Takagi et al., 2020). Compared to the wild-type (WT) PoSaV

202 Cowden strain, tissue culture-adapted (TC) PoSaV has six conserved amino acid substitutions

203 in the capsid protein (Lu et al., 2016). Four of the six amino acid substitutions in VP1 (residues

204 C178S, Y289H, M324I, and E328G) are critical for the cell culture adaptation of the PoSaV

205 Cowden strain. Although reversion of the mutations at the other two substitutions in VP1

206 (residues 291 and 295) from that of the TC strain to that of the WT reduced viral replication in

207 vitro, the revertants enhanced viral replication in vivo and induced higher-level serum and

208 mucosal antibody responses than those induced by the TC PoSaV Cowden strain (Lu et al.,

209 2016). We mapped these four essential and two functional mutations on the atomic structure of

$210 \mathrm{HuSaV}$. The corresponding residues for the six tissue culture-adapted mutations were S186,

211 H298, Y300, R304, L334, and M338 (black arrowheads in Figure S5). Except for one residue

212 S186 in the S-domain, the other five residues are exposed to the outer environment and are

213 located on the receptor-accessible surface (Figure 8), suggesting that these residues in the P

214 domain are actually involved in the receptor binding in the PoSaV. However, as the six residues

215 are not conserved in $\mathrm{HuSaV}$ (black arrows in Figure 4), the receptor molecule of HuSaV might

216 be different from that of PoSaV. Further studies are required to elucidate the receptor

217 recognition mechanism of the $\mathrm{SaV}$ based on the atomic structure of HuSaV VP1. 


\section{Acknowledgements}

221 This work was supported by the Platform Project for Drug Discovery, Informatics, and Structural

222 life Science (PDIS) from the Ministry of Education, Culture, Sports, Science and Technology

223 (MEXT), the Japan Agency for Medical Research and Development (AMED) for Supporting

224 Drug Discovery and Life Science Research (Basis for Supporting Innovative Drug Discovery

225 and Life Science Research (BINDS)) (Grant No. JP18am0101072 (support number 0194 ) to

226 N.M. and K.I.), AMED (Grant No. 20fk0108121h0402 and 16fk0108304h2003 to Ka.M. and

227 Grant No. 16fk0108304j9003 to K.K., Grant No. 16fk0108304j0203 to T.O.), MEXT KAKENHI

228 (Grant No. JP16H00786 to Ka.M.), and the collaborative programs for National Institute for

229 Physiological Sciences (to K.K.).

\section{Author Contributions}

232 N.M., K.K. and Ka.M. conceived the project. T.O., Ko.M, and K.K. and M.M. expressed the VP1

233 protein of $\mathrm{HuSaV}$ and purified the HuSaV VLPs. N.M. and Ka.M. prepared cryo-EM grids and

234 checked them at 200kV cryo-EM. N.M. and K.I. collected final high resolution cryo-EM images

235 using $300 \mathrm{kV}$ cryo-EM. N.M. processed the EM data and reconstructed the final EM map. N.M.

236 built and refined the atomic model. N.M. and Ka.M. analyzed the structure. All authors wrote the

237 paper and contributed to experimental design and wrote the manuscript. 


\section{Declaration of Interests}

240 The authors declare no competing financial interest.

\section{References}

244 Adams, P.D., Afonine, P.V., Bunkoczi, G., Chen, V.B., Davis, I.W., Echols N., Headd, J.J.,

248 Atmar R.L. and Estes, M.K. (2001). Diagnosis of noncultivatable gastroenteritis viruses, the human caliciviruses. Clin. Microbiol. Rev. 14, 15-37.

250 Chen, R., Neill, J.D., Estes, M.K., and Prasad, B.V.V. (2006). X-ray structure of a native

253 Chen, R., Neill, J.D., Noel, J.S., Hutson, A.M., Glass, R.I., Estes, M.K., and Prasad, B.V.V.

254 (2004). Inter- and intragenus structural variations in caliciviruses and their functional 
256 Chen, V.B., Arendall W.B. 3rd, Headd, J.J., Keedy, D.A., Immormino, R.M., Kapral, G.J.,

257 Murray, L.W., Richardson, J.S., and Richardson, D.C. (2010). MolProbity: all-atom structure validation for macromolecular crystallography. Acta Crystallogr. D 66, 12-21.

259 Clarke I.N., and Lambden P.R. (2000). Organization and expression of calicivirus genes. J. Infect. Dis. 181 Suppl. 2, S309-316.

261 Conley, M.J., McElwee, M., Azmi, L., Gabrielsen, M., Byron, O., Goodfellow, I.G., and Bhella, Nature 565, 377-381.

264 Emsley, P., Lohkamp, B., Scott, W.G., and Cowtan, K. (2010). Features and development of Coot. Acta Crystallogr. D 66, 486-501.

266 Farkas, T., Zhong, W.M., Jing, Y., Huang, P.W., Espinosa, S.M., Martinez, N., Morrow, A.L., Ruiz-Palacios, G.M., Pickering, L.K. and Jiang, X. (2004). Genetic diversity among sapoviruses. Arch. Virol. 149, 1309-1323.

Goddard, T.D., Huang, C.C., Meng, E.C., Pettersen E.F., Couch, G.S., Morris, J.H., and Ferrin, T.E. UCSF ChimeraX - Meeting modern challenges in visualization and analysis. Protein Sci. 27, 14-25.

272 Gouet, P., Robert, X., and Courcelle, E. (2003). ESPript/ENDscript: extracting and rendering sequence and 3D information from atomic structures of proteins. Nucl. Acids Res. 31, 3320-3323. 
275 Grigorieff, N., and Harrison, S.C. (2011). Near-atomic resolution reconstruction of icosahedral

276 viruses from electron cryo-microscopy. Curr. Opin. Struct. Biol. 21, 265-273.

277 Guu T.S.Y., Liu, Z., Ye, Q., Mata, D.A., Li, K., Yin, C., Zhang, J., and Tao, Y.J. (2009) receptor binding. Proc. Natl. Acad. Sci. U S A 106, 12992-12997.

Iwakiri, A., Ganmyo, H., Yamamoto, S., Otao, K., Mikasa, M., Kizoe, S., Katayama, K., Wakita, identification of nucleotide substitutions in the capsid protein during prolonged excretion. Arch. Virol. 154, 689-693. sapovirus-like particles. Microbiol. Immunol. 56, 760-770. 
Lu, Z., Yokoyama, M., Chen, N., Oka, T., Jung, K., Chang, K.-O. Annamalai, T., Wang, Q., and Saif, L.J. (2016). Mechanism of cell culture adaptation of an enteric calicivirus, the porcine sapovirus cowden strain. J. Virol. 90, 1345-1358.

Matsuura, Y., Tohya, Y., Mochizuki, M., Takase, K., and Sugimura, T. (2001). Identification of conformational neutralizing epitopes on the capsid protein of canine calicivirus. J. Gen. Virol. 82, 1685-1702.

Miyazaki, N., Taylor, D.W., Hansman, G.S., and Murata K. (2016). Antigenic and cryo-electron microscopy structure analysis of a chimeric sapovirus capsid. J. Virol. 90, 2664-2675.

Oka, T., Lu, Z., Phan, T., Delwart, E.L., and Saif, L.J. (2016). Genetic characterization and classification of human and animal sapoviruses. PLoS ONE 11, e0156373.

Oka, T., Wang, Q., Katayama, K., and Saif, L.J. (2015). Comprehensive review of human sapoviruses. Clin. Microbiol. Rev. 28, 32-53. receptor. J. Virol. 84, 5550-5564.

310 Pettersen, E.F., Goddard, T.D., Huang, C.C., Couch, G.S., Greenblatt, D.M., Meng, E.C., and Ferrin, T.E. (2004). UCSF Chimera - A visualization system for exploratory research and

313 Prasad, B.V.V., Hardy, M.E., Dokland, T., Bella, J., Rossmann, M.G., and Estes, M.K. (1999). 
315 Risler, J.L., Delorme, M.O., Delacroix, H., and Henaut, A. (1988). Amino acid substitutions in

316 structurally related proteins. A pattern recognition approach. Determination of a new and

317 efficient scoring matrix. J. Mol. Biol. 204, 1019-1029.

318 Rohou, A. and Grigorieff, N. (2015). CTFFIND4: Fast and accurate defocus estimation from

319 electron micrographs. J. Struct. Biol. 192, 216-221.

320 Rossmann M.G. and Johnson, J.E. (1989) Icosahedral RNA virus structure. Annu. Rev. Biochem. $58,533-573$.

322 Scheres, S.H. (2012). RELION: implementation of a Bayesian approach to cryo-EM structure determination. J. Struct. Biol. 180, 519-530.

324 Song, C., Takai-Todaka, R, Miki, M., Haga, K., Fjimoto, A., Ishiyama, R., Oikawa, K.,

Takagi, H., Oka, T., Shimoike, T., Saito, H., Kobayashi, T., Takahashi, T., Tatsumi, C., Kataoka, cell lines supplemented with bile acids. Proc. Natl. Acad. Sci. U S A, online ahead of print. Doi: $10.1073 /$ pnas.2007310117.

332 Tang, G., Peng, L., Baldwin, P.R., Mann, D.S., Jiang, W., Rees, I., and Ludtke, S.J. (2007). 
335 Thompson, J.D., Higgins, D.G., and Gibson, T.J. (1994). CLUSTAL W: improving the sensitivity of progressive multiple sequence alignment through sequence weighting, position-specific gap penalties and weight matrix choice. Nucleic Acids Res. 22, 4673-4680.

Tohya, Y., Masuoka, K., Takahashi, E., and Mikami, T. (1991). Neutralizing epitopes of feline calicivirus. Arch. Virol. 117, 173-181.

Wang, X., Xu, F., Liu, J., Gao, B., Liu, Y., Zhai, Y., Ma, J., Zhang, K., Baker, T.S., Schulten, K., Zheng, D., Pang, H., and Sun, F. (2013). Atomic model of rabbit hemorrhagic disease virus by cryo-electron microscopy and crystallography. PLoS Pathog. 9, e1003132.

344 Xing, L., Li, T.-C., Mayazaki, N., Simon, M.N., Wall, J.S., Moore, M., Wang, C.-Y., Takeda, N., the crystal structure. Proc. Natl. Acad. Sci. U S A 103, 12986-12991.

Yinda, C.K., Conceição-Neto, N., Zeller, M., Heylen, E., Maes, P., Ghogomu, S.M., Ranst, M.V., and Matthijnssens, J. (2017). Novel highly divergent sapoviruses detected by 
354 metagenomics analysis in straw-colored fruit bats in Cameroon. Emerg. Microbes Infect. 6,

$355 \quad$ e38.

356 Zhang, K. (2017). http://www.mrc-lmb.cam.ac.uk/kzhang/ . 
Methods

A baculovirus expression system constructed in a previous study (Kitamoto et al., 2012) was

364 infect approximately $3 \times 10^{6}$ confluent Hi5 cells (Thermo Fischer Scientific, USA) at a

365 multiplicity of infection (MOI) of 5-10 in 1.5 mL Ex-Cell 405 medium (Merck, Germany), and

366 the infected cells were incubated at $26^{\circ} \mathrm{C}$. The culture medium was harvested 5-6 days

367 post-infection (dpi), centrifuged for $10 \mathrm{~min}$ at 3,000 $\times \mathrm{g}$, and further centrifuged for $30 \mathrm{~min}$ at

$36810,000 \times \mathrm{g}$. The VLPs were concentrated by ultracentrifugation for $2 \mathrm{~h}$ at $31,000 \mathrm{rpm}$ at $4{ }^{\circ} \mathrm{C}$

369 (Beckman SW-31Ti rotor), and then resuspended in $500 \mu \mathrm{L}$ of Ex-Cell 405 medium. Samples

370 were examined for VLP formation by conventional electron microscopy after the VLPs were

371 purified by $\mathrm{CsCl}$ as described previously (Miyazaki et al., 2016).

\section{Cryo-electron microscopy (cryo-EM) data collection and processing}

374 For cryo-EM experiments, $3 \mu \mathrm{L}$ of sample solution was applied to a Quantifoil holey carbon

375 grid (R1.2/1.3, Mo 200 mesh, Quantifoil Micro Tools $\mathrm{GmbH}$ ) at $4{ }^{\circ} \mathrm{C}$ with $100 \%$ humidity, and

376 then plunge-frozen into liquid ethane using a Vitrobot Mark IV (Thermo Fisher Scientific, USA).

377 The cryo-EM grids were examined at liquid nitrogen temperature using a cryo-electron 
378 microscope (Titan Krios, Thermo Fisher Scientific), incorporating a field emission gun and a

379 Cs-corrector (Corrected electron optical systems $\mathrm{GmbH}$ ). The microscope was operated at 300

$380 \mathrm{kV}$ and a nominal magnification of $\times 75,000$. Movie frames were recorded using a Falcon II

381 direct electron detector (Thermo Fisher Scientific), applied with a nominal underfocus value

382 ranging from -1.0 to $-2.5 \mu \mathrm{m}$. An accumulated dose of 20 electrons per $\AA^{2}$ on the sample was

383 fractionated into a move stack of 16 image frames with $0.0625 \mathrm{~s}$ per frame, for a total exposure

384 time of $1.0 \mathrm{~s}$. The workflow of the cryo-EM image processes is summarized in Figure S1B.

385 Movies $(0.87 \AA$ / pixel) were subsequently aligned and summed using MotionCorr software (Li et

386 al., 2013) to obtain a final motion-corrected image. Estimation of the contrast transfer function

387 was performed using the CTFFIND program (Rohou and Grigorieff, 2015). Micrographs

388 exhibiting poor power spectra (based on the extent and regularity of the Thong rings) were

389 rejected (4.0 Å resolution cutoff). Approximately 2,000 particles were manually picked using

390 EMAN2 (Tang et al., 2007) and used to generate 2D classes for templates for auto-picking in

391 Gautomatch (Zhang, 2017; K. Zhang, MRC Laboratory of Molecular Biology, Cambridge, UK,

392 http://www.mrc-lmb.cam.ac.uk/kzhang/Gautomatch). All the following processes were

393 performed using RELIOIN (Scheres, 2012). 79,147 auto-picked particles from 2,918

394 micrographs were subjected to reference-free 2D classification. A total of 77,352 particles were

395 selected from acceptable 2D classes (Figure S1C) and were then subjected to two rounds of 3D

396 classification with icosahedral symmetry. Finally, the 3D structure was reconstructed from

39723,434 particles at $2.9 \AA$ resolution, which was estimated by the gold standard FSC with a 0.143 
398 cutoff (Grigorieff and Harrison, 2011). The local resolution variations were also calculated using

399 the RELION software (Figure S2).

$401 \quad$ Atomic model building and three-dimensional homology mapping

402 The $2.9 \AA$ map was used for de novo atomic model construction of the VP1 protein in O

403 (Jones et al., 1991). The initial atomic model was refined with phenix.real_space_refine (Adams

404 et al., 2010) and manual adjustment in COOT (Emsley et al., 2010). The final model was further

405 validated using MolProbity (Chen et al., 2010). The sequences of SaV VP1 proteins were aligned

406 using CLUSTAL-W (Thompson et al., 1994). Identical and similar amino acid residues were

407 defined according to the Risler matrix (Risler et al., 1988) and were mapped onto the surface of

408 the SaV VP1 protein from GI. Nichinan using UCSF Chimera and ChimeraX software (Pettersen

409 et al., 2004; Goddard et al., 2018).

\section{Data availability}

412 The cryo-EM map of the HuSaV VLP of the Nichinan strain has been deposited in the Electron

413 Microscopy Data Bank under accession number EMD-30793. Atomic coordinates for the atomic

414 model of the VLP have been deposited in the Protein Data Bank under accession number 7DOD. 


\section{$417 \quad$ Figures}

\section{A}
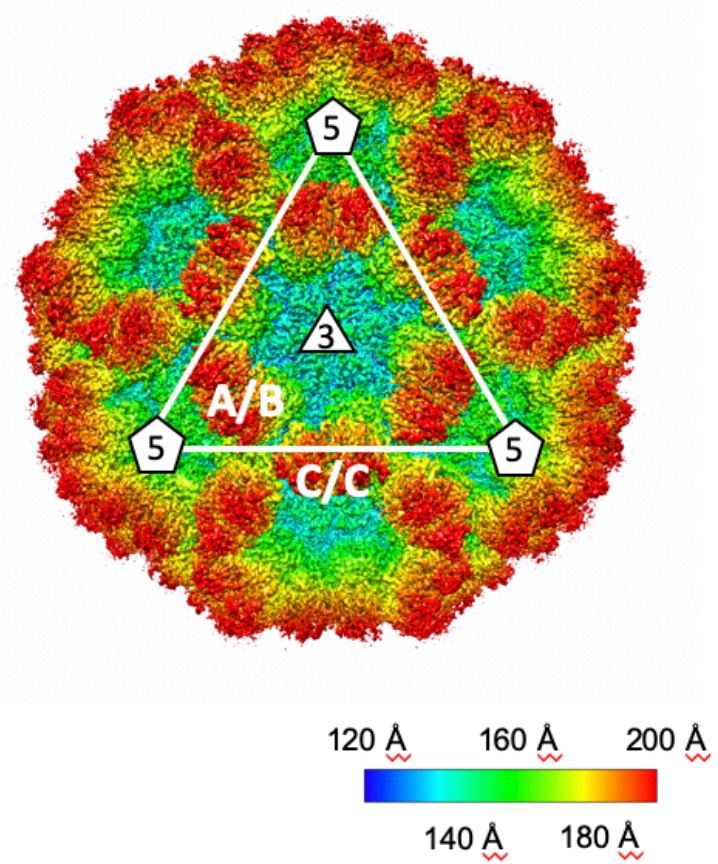

Radial distance
B

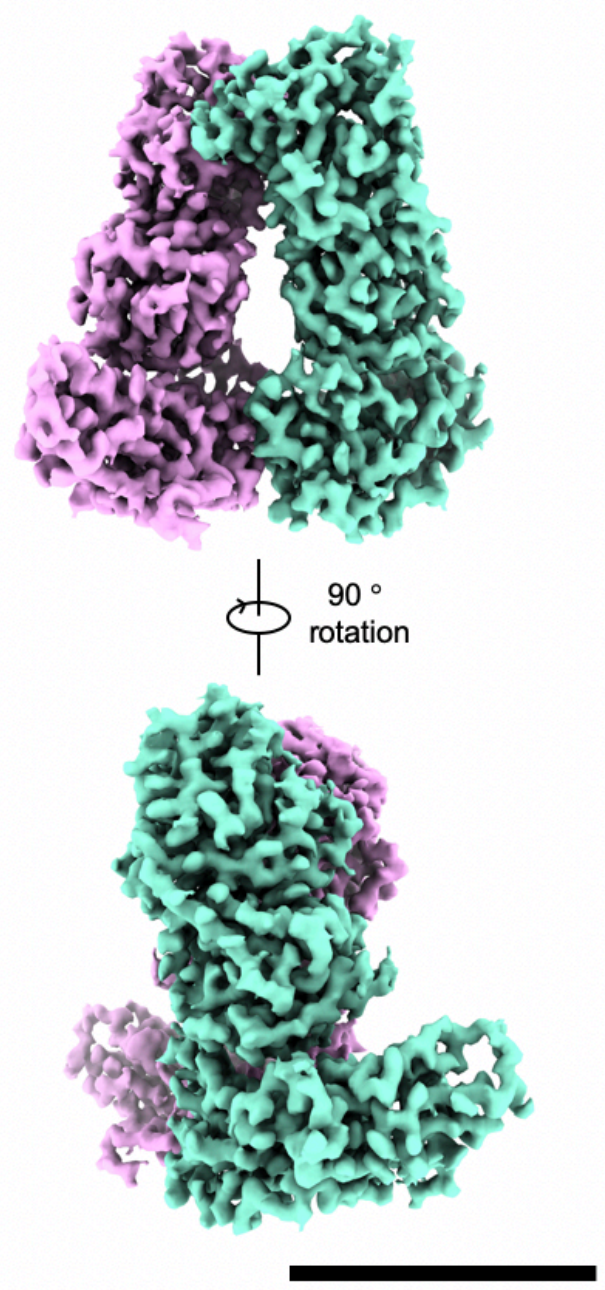

419 Figure 1. Cryo-EM maps of the HuSaV-VLP. A) Surface representation of the cryo-EM map

420 colored according to the distance from the center of the particle. Scale bar: $10 \mathrm{~nm}$. B) Surface

421 representation of the cryo-EM map of a HuSaV VP1 C/C dimer. Monomers are colored light

422 green and pink. Scale bar: $5 \mathrm{~nm}$.

423 


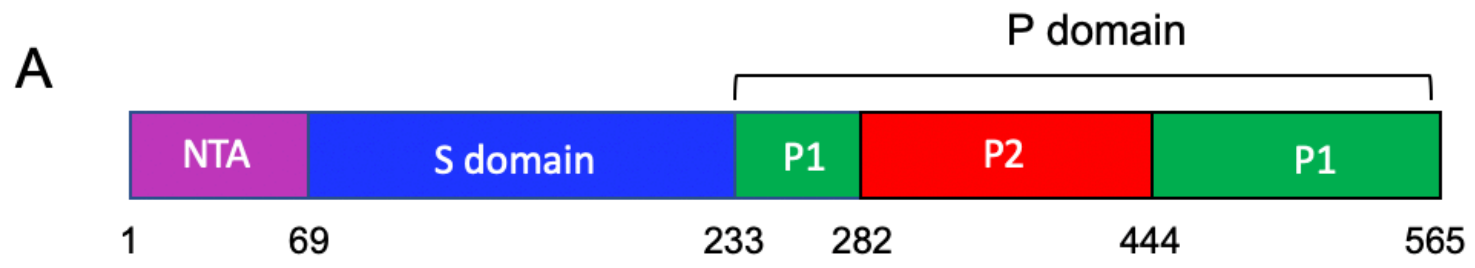

B

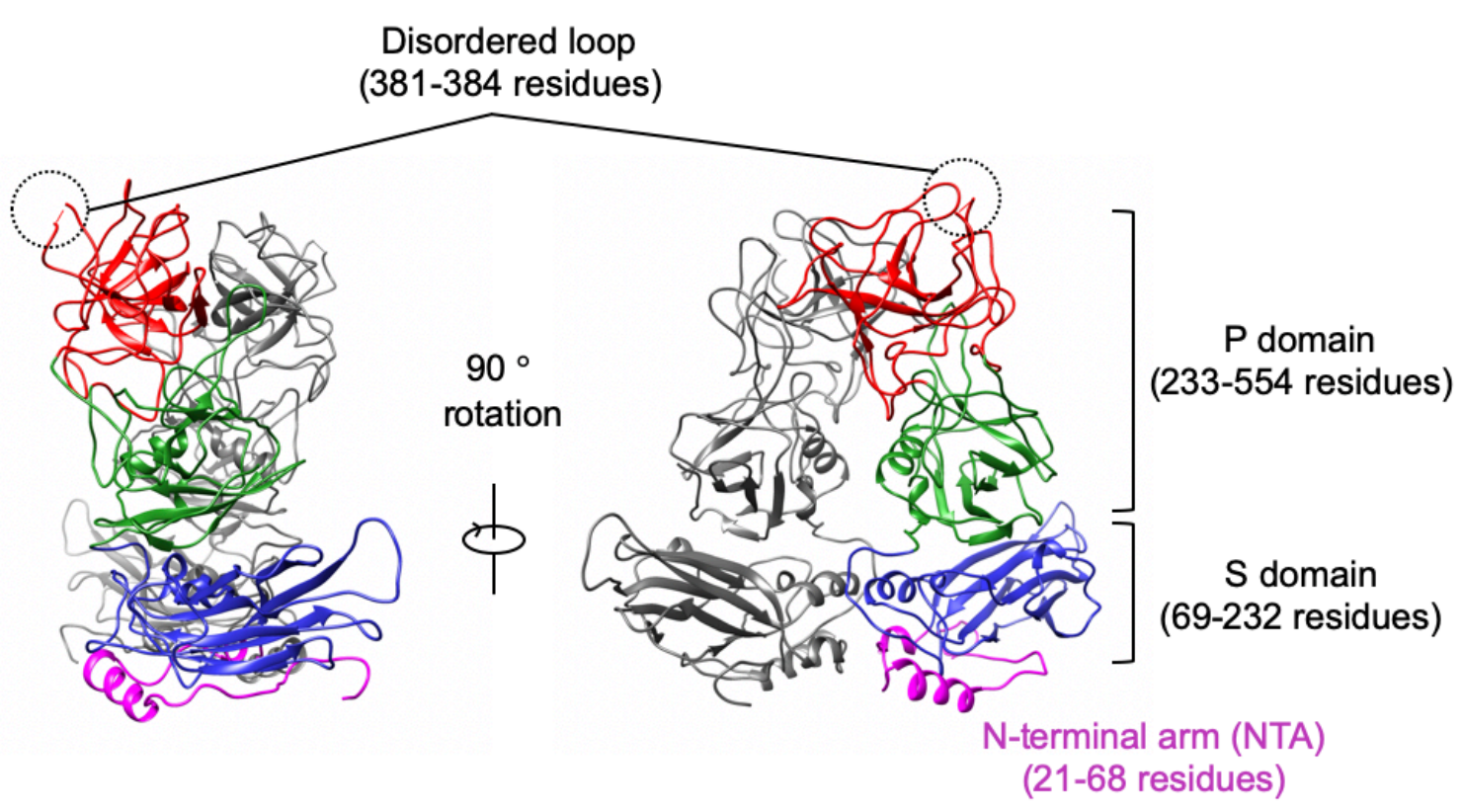

C

Disordered loop
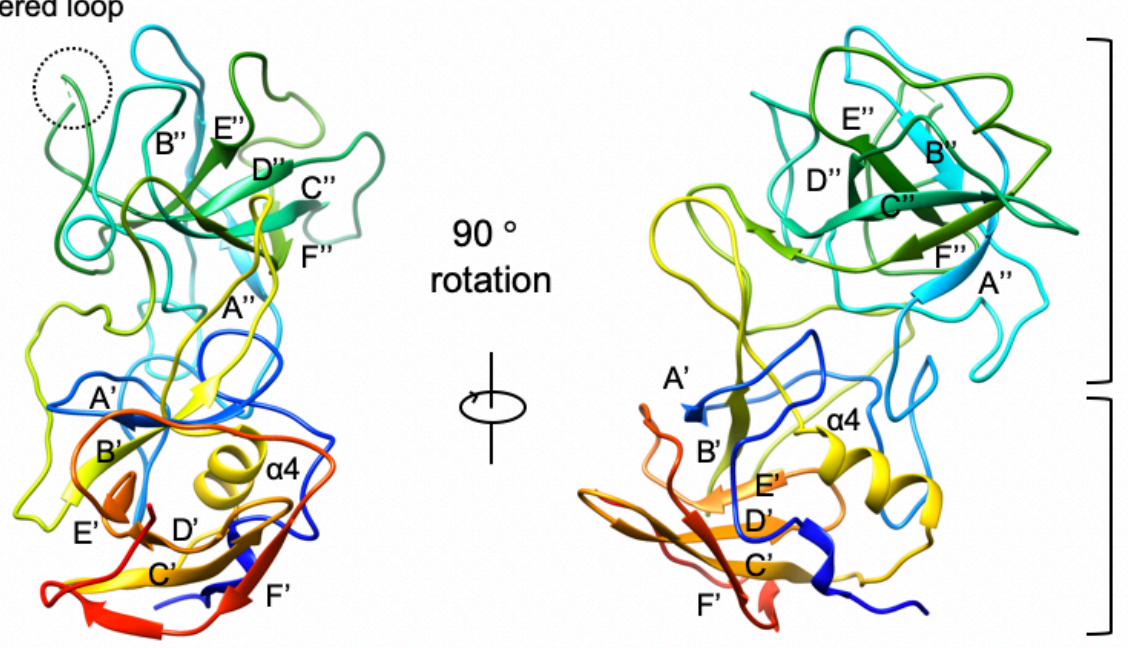

P2 domain

P1 domain

425 Figure 2. Atomic structure of the HuSaV VP1. A) Domain organization of the HuSaV VP1.

426 N-terminal arm (NTA), S domain, P1, and P2 subdomains are colored magenta, blue, green, and 
427 red, respectively. B) Ribbon drawing of a $\mathrm{HuSaV} \mathrm{C/C} \mathrm{dimer.} \mathrm{An} \mathrm{image} \mathrm{(right)} \mathrm{is} \mathrm{drawn} \mathrm{after} \mathrm{the}$

428 rotation of the left image by $90^{\circ}$. A disordered loop (residues 381-384) on the capsid surface is

429 highlighted by a dotted circle. NTA, S domain, P1, and P2 subdomains in a monomer are colored

430 similar to (A). C) Ribbon representation of a HuSaV P-domain. The P domain is rainbow colored

431 with the N-terminus in blue and the C-terminus in red. An image (right) is drawn after the

432 rotation of the left image by $90^{\circ}$. All secondary-structural elements are labelled. 

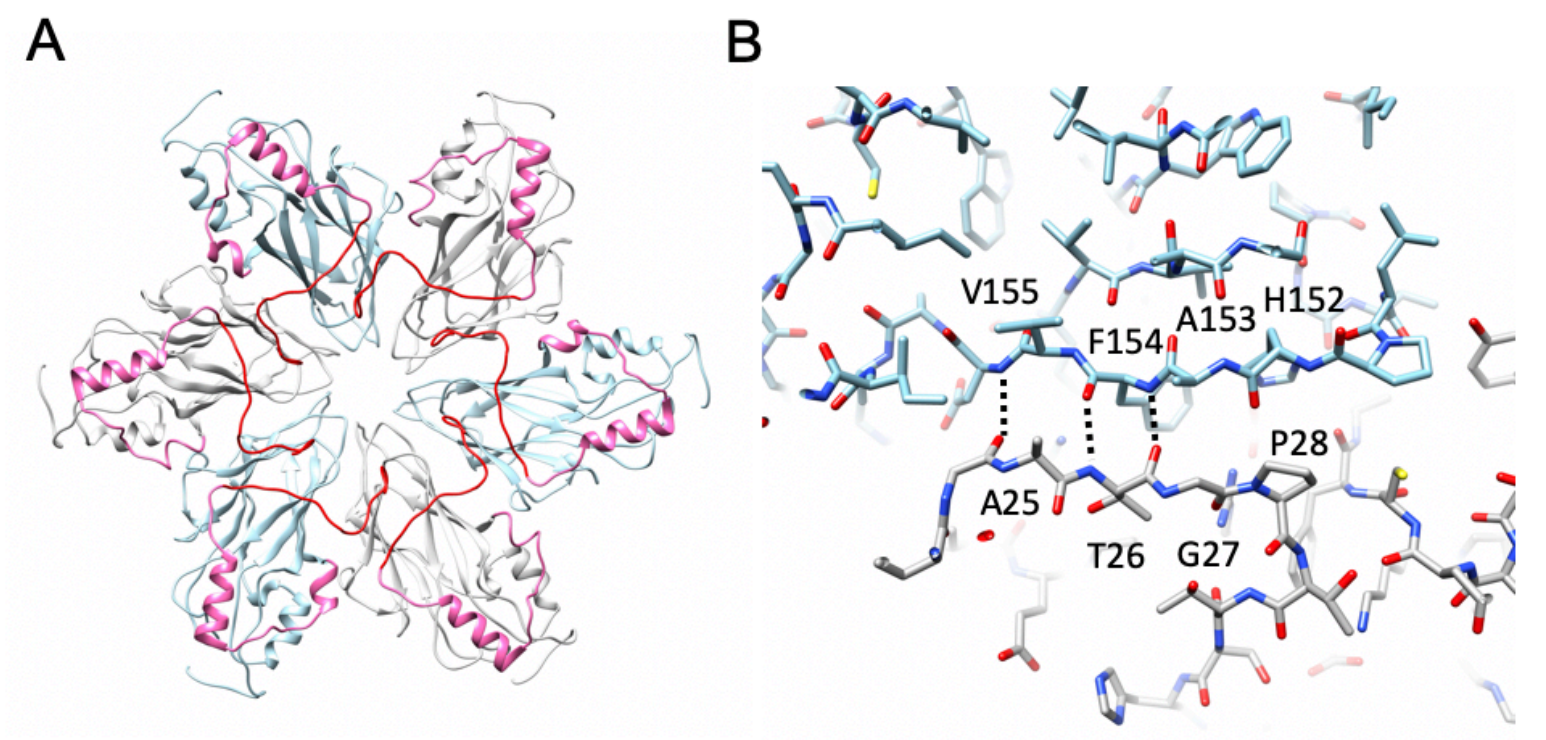

435 Figure 3. N-terminal arm network of VP1. A) Ribbon representation of S-domains around an

436 icosahedral 3-fold axis viewed from the inside of the particle. S-domains of the B-and C-subunit

437 are shown in gray and light blue, respectively. The N-terminal arms are highlighted by red

438 (residues 22-37) and pink (residues 38-68). B) Inter-subunit $\beta$-sheet formed by the N-terminal

439 arm. B- and C-subunits are shown in gray and light blue, respectively. 
bioRxiv preprint doi: https://doi.org/10.1101/2021.02.09.429995; this version posted February 9, 2021. The copyright holder for this preprint

(which was not certified by peer review) is the author/funder, who has granted bioRxiv a license to display the preprint in perpetuity. It is made available under aCC-BY 4.0 International license.

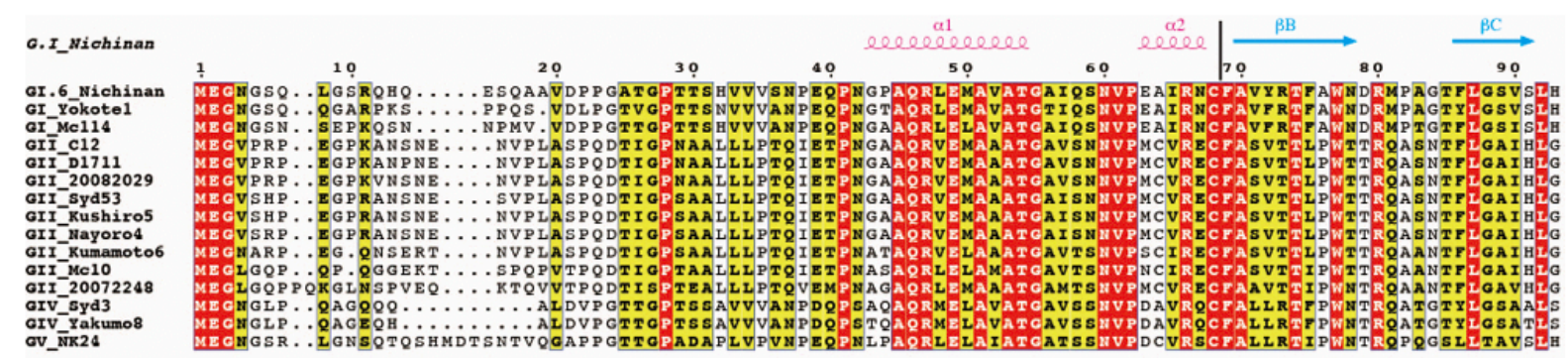

G.I_Michinan

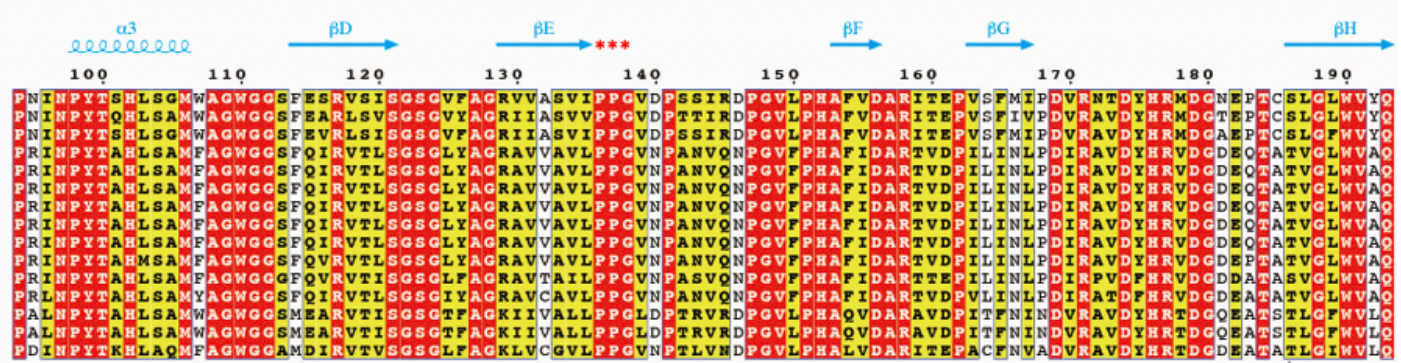

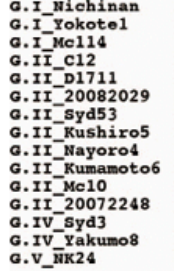

G.I_Nichinan

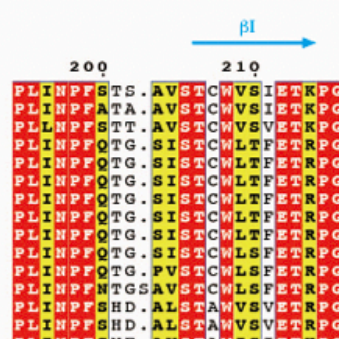

G.I_Hichinan

G.I Mc114

G.II-D1711

G.II-20082029

G.II-Kushiro5

G.II-20072248

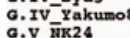

G.I_Nichinan

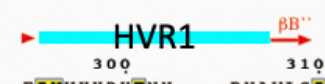

220

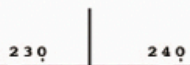

250

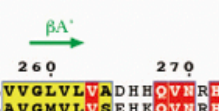

${ }_{280}^{* * *} \underbrace{}_{290} \frac{\beta \mathrm{A}}{0^{*}}$

G.I_Hichinan

G.I Mokote

G.II_c12

G.II-20082029

G.II_Kushiro

G.II_Nayoro4

G.II-Mo10

c.rv_syd3

G.v_ñK24

20

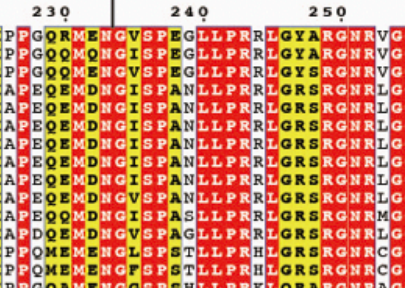

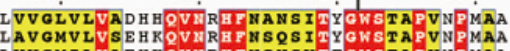

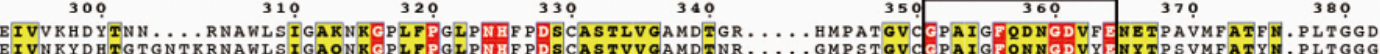
TH 作 AISWYQNTTPG... . ISTRGLLSAEGKGIIFPNIVMBWTDVALSSKTSGRTTVPTDQANLNQCPGASOPVVMFQNMGDVNETSANNCVLTAAS. . . BDFV

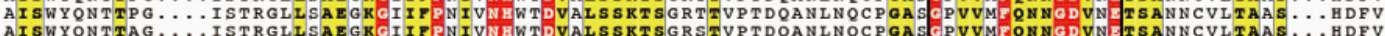
AISWYQNTTAG.... ISTRGLLSAEGK

AISWYQNTTAG... ITSTRGLISAEG

DISWYGDAGN..... KSIRGLVSAQG

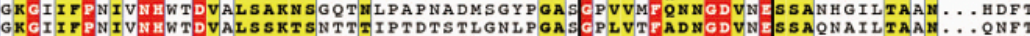

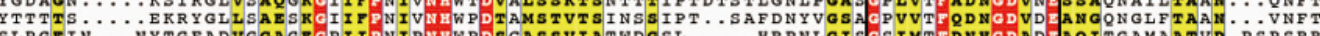

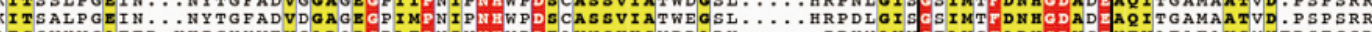
\

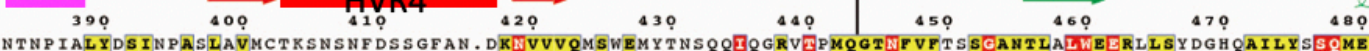

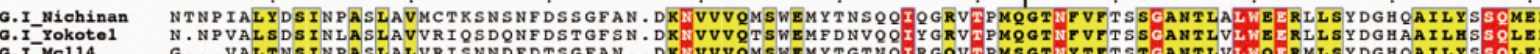
G.IMc114

G.IT_C12

G.II-20082029

G.II-Kushiro5

c.II-Kumamoto

G.II-200722

G. Iv -Syd3

W

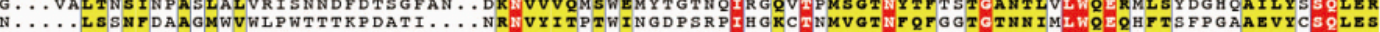

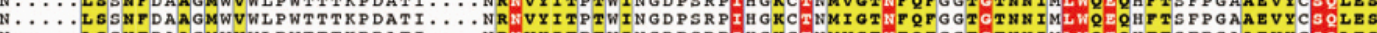

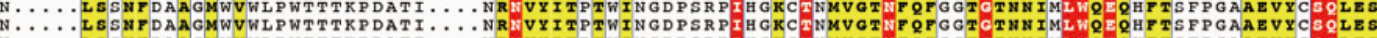

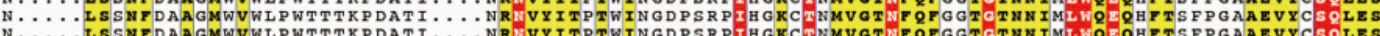

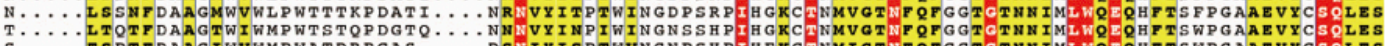

... DSEIYISPTWVNGNPSHPIHEKCTNMIGTNFQFG GTGTHNIMLWRERHFTSWPGAAEVYCSQLES

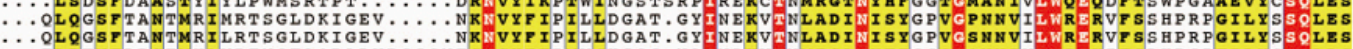

G.v_üK24

G.I_Nichinan

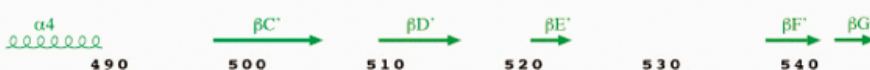

G.I_Hichinan

G.I Yokotel

G.III-C12

G.II-20082029

G.II_Kushiros

G.II_Nayoro4

G.IIIMc10

G.Iv-syd3

c. Iv yaku
G.v_uK24

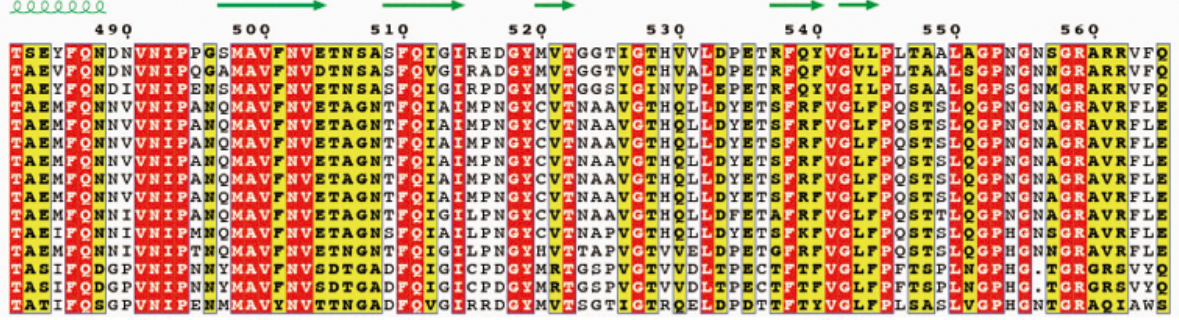




\section{Figure 4. Alignment of the amino acid sequences of the HuSaV VP1 proteins. The}

443 secondary-structural elements are indicated over the sequences as a spiral ( $\alpha$-helix) or an arrow

444 ( $\beta$-sheet) and are colored according to the domain regions (S: pink, P1: green, P2: orange). The

445 regions of the $\mathrm{S}, \mathrm{P} 1$, and $\mathrm{P} 2$ domains are also shown as pink, green, and orange lines below the

446 secondary structural elements, respectively. Letters on a red and yellow background indicate

447 identical and similar amino acids based on a Risler matrix (Risler et al., 1988), respectively.

448 Gaps are shown as dotted lines. Figure is drawn by ESPript (Gouet et al., 2003). 
A

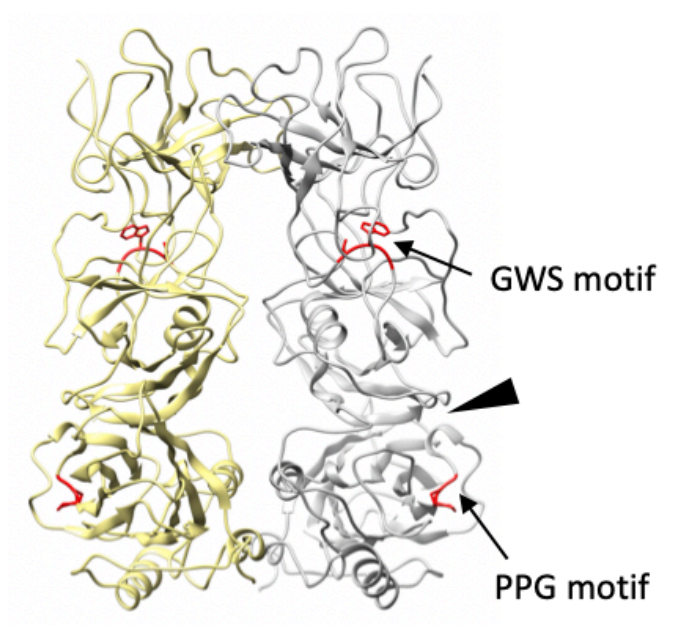

B

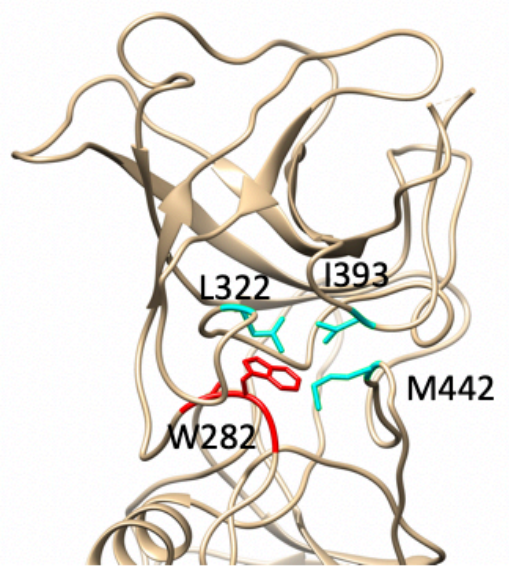

C

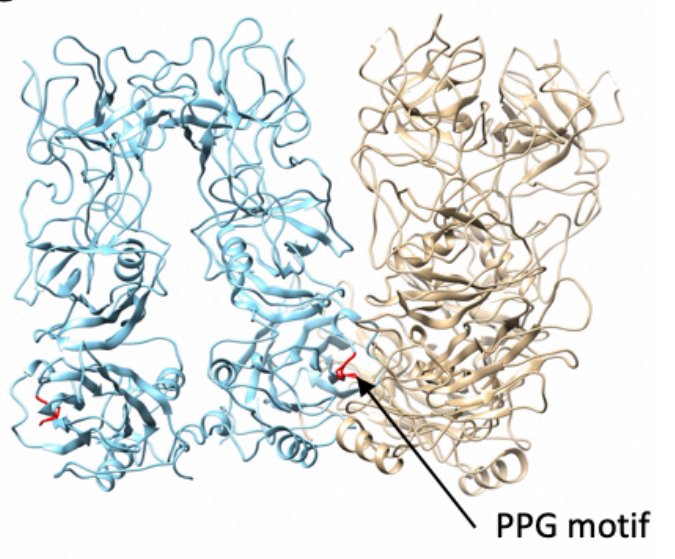

$f-\stackrel{90^{\circ}}{\text { rotation }}$

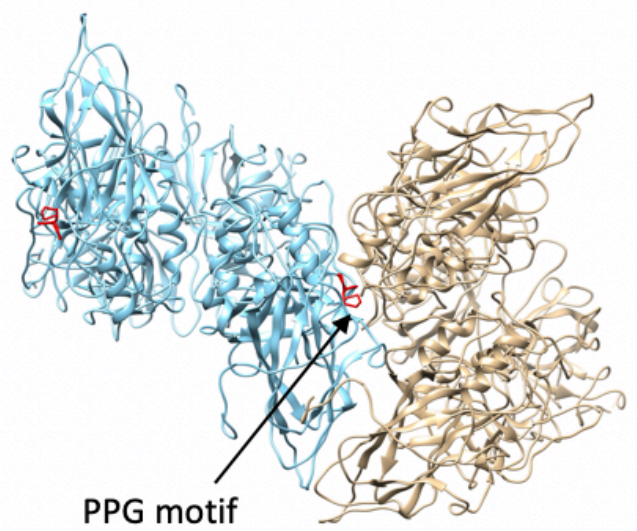

451 Figure 5. Conserved motifs among HuSaV VP1 proteins. A) Ribbon representation of a

452 HuSaV VP1 dimer showing two well-conserved motifs in the HuSaV VP1 protein, "PPG"

453 (residues 136-138) and "GWS" (residues 281-283), highlighted by red. An arrowhead indicates

454 the interaction between the loop following the PPG motif and P1 subdomain. B) The "GWS"

455 motif exists at the domain boundary between the P1 and P2 subdomains, and W282 interacts

456 with L322, I393, and M442 in the P2 subdomain. C) The "PPG" motif, colored red, is located at

457 the inter-subunit interface between the S-domains. A/B and C/C dimers are colored sky-blue and

458 gold, respectively. 
A
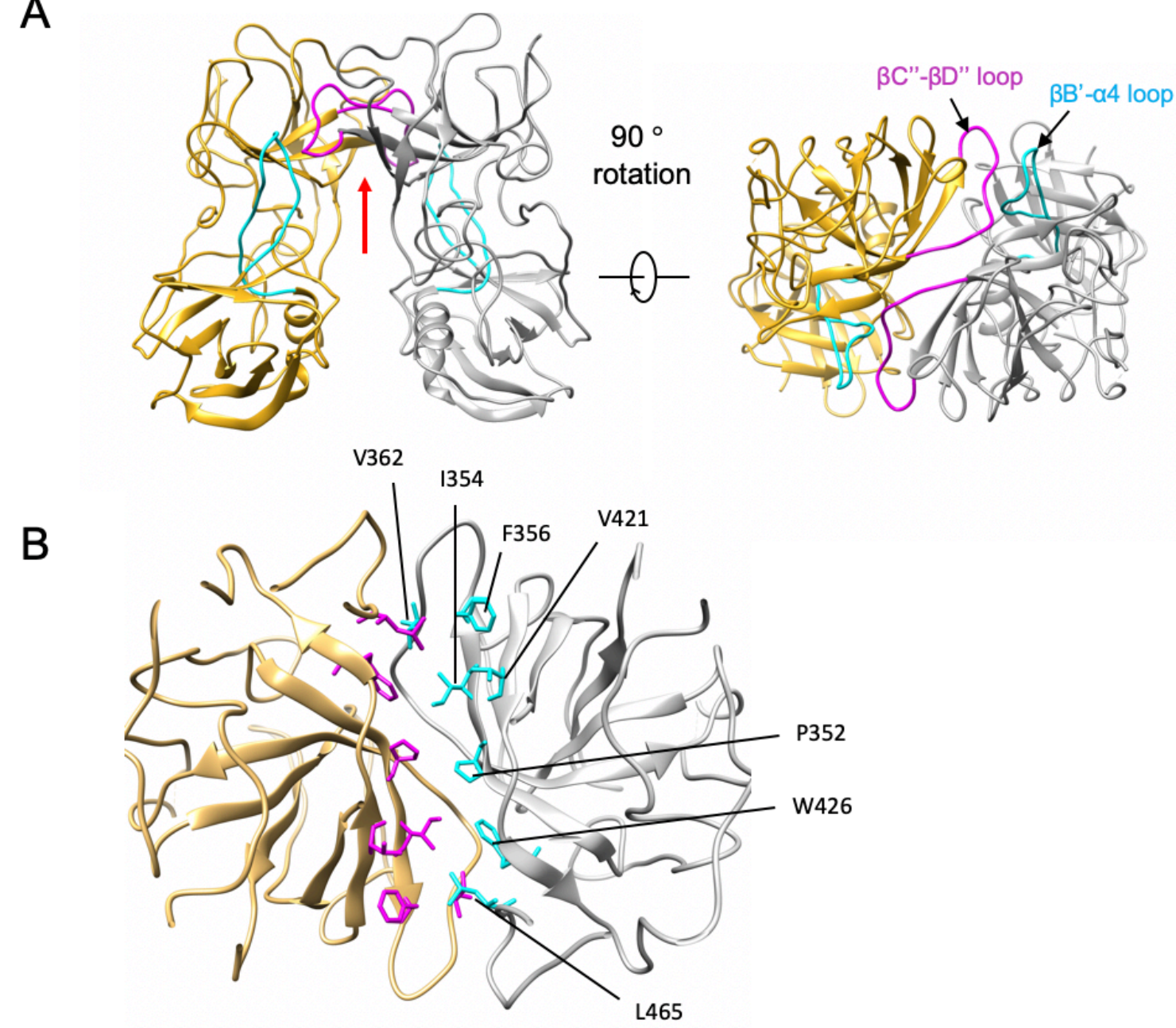

C

Buried surface area between $\mathrm{P}$ domains in dimers of caliciviruses

\begin{tabular}{ccc}
\hline & P domain residues & Buried surface area between $\mathbf{P}$ domains $\left(\AA^{2}\right)$ \\
\hline Sapovirus & $233-554$ & 1,100 \\
Norovirus & $226-520$ & 1,500 \\
Vesivirus (SMSV) & $362-703$ & 1,700 \\
Lagovirus & $236-569$ & 1,500 \\
\hline
\end{tabular}

460

461 
462 Figure 6. Dimeric interaction of P-domains. A) Ribbon drawing of a HuSaV P-domain dimer.

463 Two loops, $\beta C$ " $-\beta \mathrm{D}$ ” and $\beta \mathrm{B}$ '- $\alpha 4$ loops, are highlighted by magenta and cyan, respectively. B)

464 Hydrophobic interactions at the dimer interface viewed from the direction indicated by a red

465 arrow in (A). C) Buried surface area between P domains in the dimers of caliciviruses. 
bioRxiv preprint doi: https://doi.org/101101/2021.02 09.429995. this version posted February 92021 . The copyright holder for this preprint (which was not certified by peer review) is the author/funder, who has granted bioRxiv a license to display the preprint in perpetuity. It is made available under aCC-BY 4.0 International license.

A

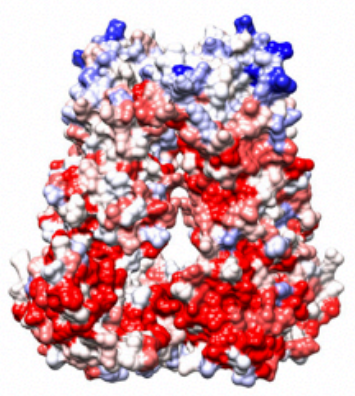

$90^{\circ}$ rotation

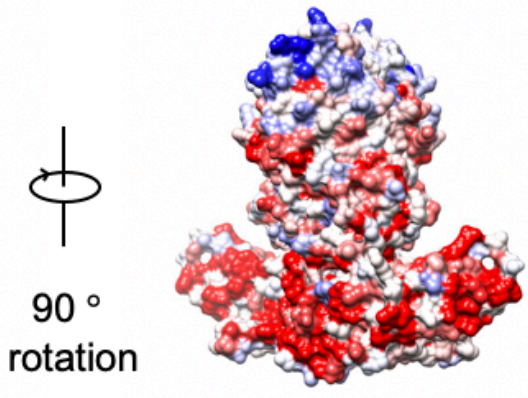

Side views

B

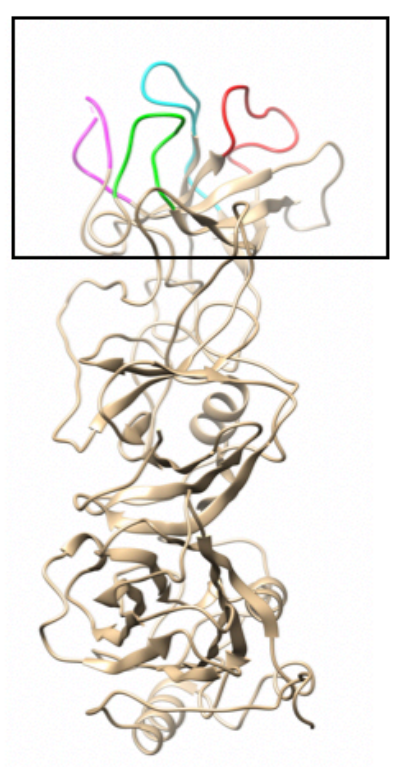

$90^{\circ}$

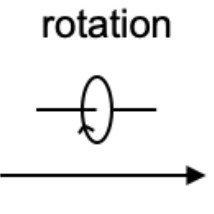

Top view

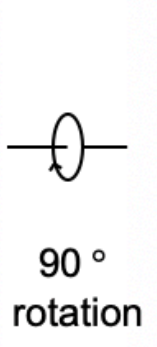

$\begin{array}{lll}0.0 & 0.5 & 1.0\end{array}$

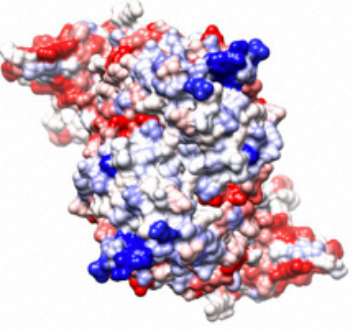

Conservation
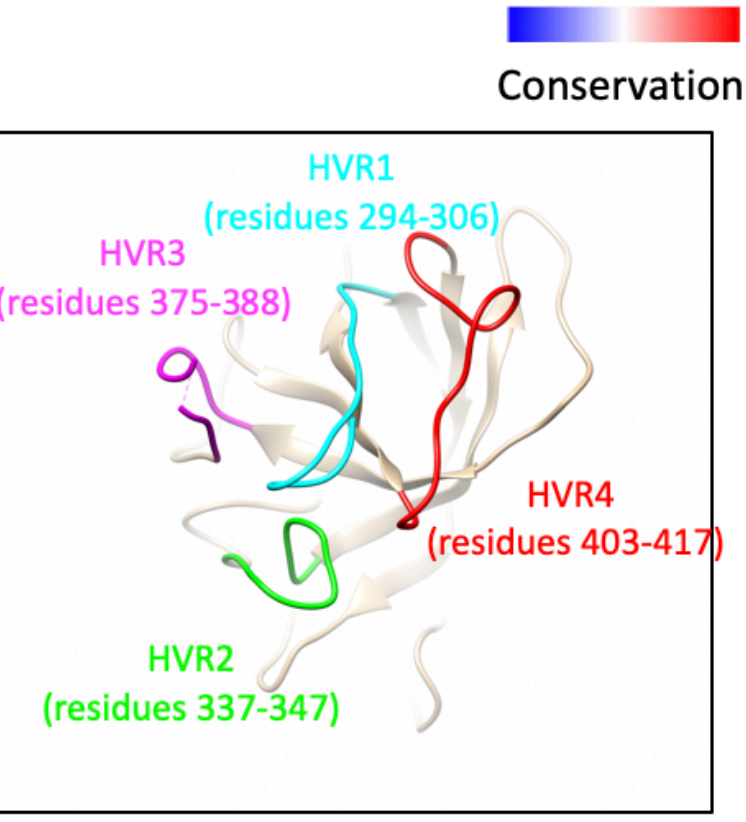

C
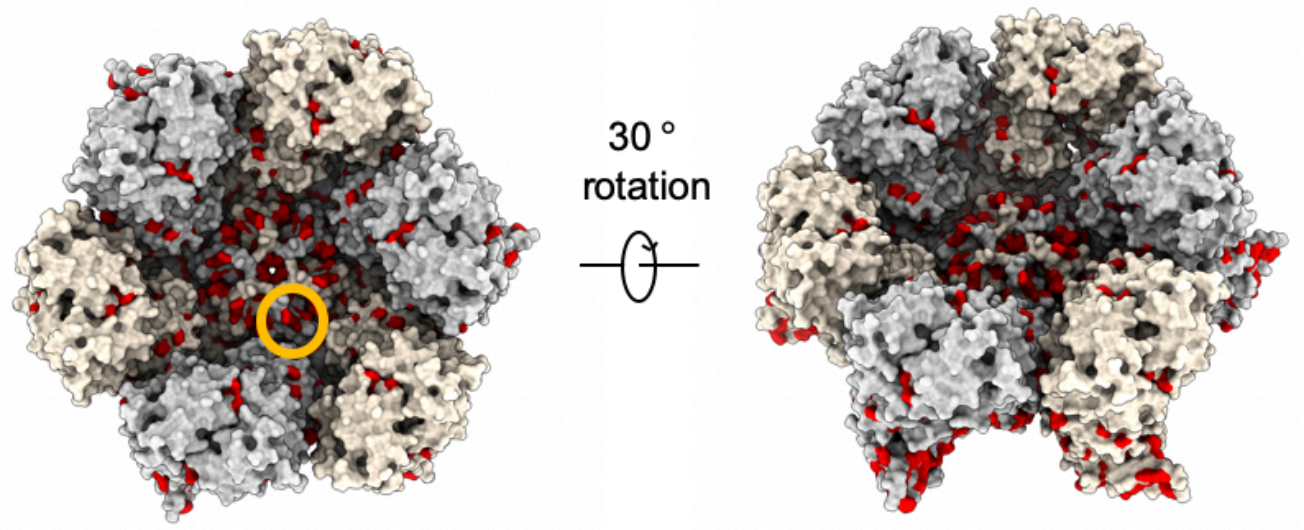
468 Figure 7. Amino acid conservation and immune-responsible surface. A) Conservation of

469 amino acid residues of HuSaV VP1 proteins mapped onto the molecular surface of a dimer. Red

470 indicates most conserved and blue indicates least conserved regions among the HuSaVs listed in

471 Figure 3. B) Hyper variable regions (HVRs) of HuSaVs. Four HVRs, HVR1 to HVR4, are

472 colored cyan, green, magenta, and red, respectively. C) Conserved amino acid residues are

473 highlighted by red on the molecular surface of the VP1 proteins around an icosahedral 6-fold

474 axis.

475 


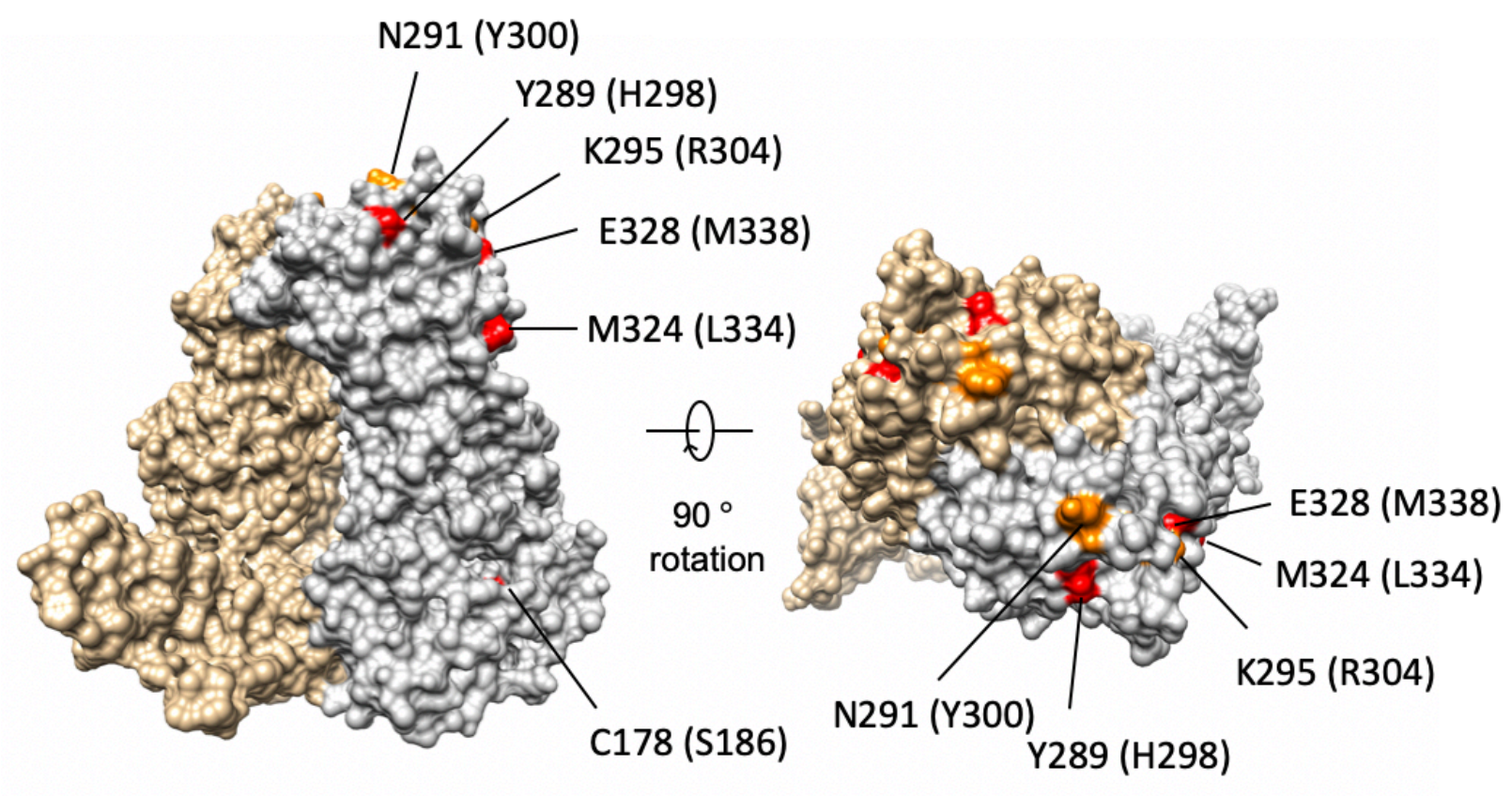

477 Figure 8. Possible receptor binding position. Six tissue culture-adapted mutation positions in

478 the wild-type PoSaV Cowden stain are mapped on a molecular surface of a VP1 dimer in a

479 Nichinan strain. Four essential and two functional mutation positions are labeled and colored red

480 and orange, respectively. The corresponding amino acid residues in the Nichinan strain is

481 enclosed in parentheses. 